\title{
Entretien avec Mr. Le Professeur Honoris Causa, Aryon Dall'Igna Rodrigues
}

\author{
Juillet 2012, Laboratoire des Langues Indigènes (LALI-IL-UnB) \\ Réalisé par Ana Helena Rossi, Professeur Adjoint, UnB-LET \\ (Traduit par Ana Helena Rossi)
}

\section{Biobibliographie}

Aryon Dall'Igna Rodrigues est né dans l'état du Paraná. En 1951, après une maîtrise en Lettres Classiques obtenue à l'Université Fédérale du Paraná, il entreprend son premier voyage de recherche chez les indiens Kaingang. Entre 1955/1955, il fait son Doctorat à Hambourg, toujours inédit au Brésil, et qui s'intitule "Phonologie der Tupinambá-Sprache ». En 1963, il est embaûché par l'Université de Brasília et devient le premier chef du premier Département de Linguistique crée dans une université brésilienne. Il installe le premier le premier Master II en Linguistique du Brésil. En 1963/1964, il dirige le premier cours intensif pour préparer des enseignants en linguistique réalisé avec le soutien financier du MEC. À l'UnB, il crée le Centre d'Études des Cultures et Langues Indigènes, et devient le Coordonnateur Général de la Pós-Graduação. En 1965, il donne sa démission pour protester contre la situation politique, et "atterrit" au Musée National (UFRJ). En 1968, il crée et coordonne le deuxième Programme de Pós-Graduação en Linguistique au Brésil, et intervient comme Chef du Secteur Linguistique du Musée National. Il negocie, alors, um fonds de la Fondation Ford pour réaliser une série de rencontres intitulées "Institut Brésilien de Linguistique", ainsi que pour former des docteurs aux États-Unis. En 1969, il participe de la création de l'Association Brésilienne de Linguistique (ABRALIN) pour lequel il avait été élu son premier Président. En 1973, il se transfert avec un groupe de professeurs et 25 póst-graduando du Musée National vers la UNICAMP dans le nouvellement créé Programme de PósGraduação em Linguística. Dans les années 1980, il participe de la création du GT Langues Indigènes, et devient son premier coordonnateur. Dans le IELUNICAMP, il est coordonnateur du Programme de Pós-Graduação. Em 1988, il prend sa retraite et reintegre la $\mathrm{UnB}$ où il consolide un domaine de recherche 
dans les langues indigènes qui gagne corps avec le LALI - Laboratoire des Langues Indigènes. ${ }^{1}$

Ana Helena Rossi (AHR) : Quelle est la relation de la linguistique avec la traduction?

Aryon Dall'Igna Rodrigues (ADR) : Si l'on entend linguistique par l'étude scientifique de la nature du langage humain, et de ses multiples manifestations dans les langues des peuples, la première relation est justement ce point commun, cette capacité humaine de communiquer à travers les langues, mais non pas seulement communiquer mais organiser la connaissance. Il ne s'agit pas seulement d'envoyer des messages à l'autre. C'est comprendre les choses. Le langage humain permet à l'humanité de comprendre. C'est le premier point du langage. Sans le langage humain comme nous avons, difficilement nous aurions toute notre base de raisonnement qui fait de l'homme être l'homme qu'il est en contraste avec le restant des êtres vivants. Nous n'avons encore aucune évidence d'une pensée élaborée par des êtres non-humains, même les plus apparentés comme les anthropoïdes. C'est une grande différence. La langue ou le langage fait cela. Il est vrai que les chimpanzés se communiquent, mais pas de manière articulée comme nous qui pouvons développer des thèmes, des expériences. C'est une grande différence qui fait de nous le fait de ne pas être des singes. Ce n'est pas simplement une communication mais l'organisation de la connaissance qui permet l'accumulation de la connaissance. Sinon on oublierait de suite. Cela est la langue, le langage. Chacune est un cas particulier du langage humain. Ce qui est notable est que malgré qu'il y ait des milliers de langues, elles ont toutes le même dénominateur commun qui est d'être l'instrument de notre cerveau avec une organisation et de l'accumulation de connaissance. Vue ainsi, nécessairement, les langues/langages sont toutes en principe traduisibles les unes vers les autres avec la restriction que les expériences humaines sont différentes. Alors, il existe des langues qui réflètent des différences dans la conception et l'explication des phénomènes du monde. Pendant que nous avons une explication pour le lever du soleil et de la lune - notre conception astronomique dit que les astres sont en relation, perception que nous avons atteint il $\mathrm{y}$ a très longtemps, mais seulement une partie de l'humanité et non pas toute l'humanité, cette conception que la terre est un objet qui tourne autour d'un autre qui est le soleil, et la lune qui est le troisième objet mais qui tourne autour de la terre appelé satellite - tous les peuples n'ont pas cette conception. La plupart des peuples indigènes ne voit pas ainsi. Cela ne veut pas dire qu'ils soient dans l'erreur.

1 Les données biographiques proviennent de la source ci-après : D’Angelis, Wilmar R., "Aryon das Línguas Rodrigues", Estudos da Linguagem, Vitória da Conquista, v.4, n.2. p. 13-19, dezembro de 2006. 
AHR : C'est une question de normativité ?

ADR : Non, c'est une question d'expérience et de perception. Ils voient la relation entre soleil et lune, et entre soleil et terre de manière différente, des fois symbolique. La lune est un animal qui à certains moment est plus vif, plus actif, de l'autre côté il s'éteint, meurt et revient de nouveau. Ce qui se réflète encore dans notre terminologie traditionnelle avec les expressions suivantes : "lune croissante", "lune décroissante". Ce sont des conceptions un peu animales, et cela n'est pas différent des autres peuples. Nous aussi, par le passé, nous avons considéré la naissance de la lune comme l'être humain qui commence à vivre. Les cultures se différencient en fonction des expériences de leurs locuteurs. Différents peuples passent par des phases semblables. Les uns s'antécipent, les autres restent arrêtés, conservateurs. En ce qui concerne l'histoire du soleil et de la lune des peuples indigènes, cela ne signifie pas qu'ils soient un peuple mythique qui vit seulement de l'imagination. C'est une vision de la réalité comme elle l'a déjà été pour nous aussi. Alors, face à cela, la traduction a un principe que tout est traduisible. Mais pour que ce soit traduisible, il faut rendre compatible des visions différentes. Traduire du portugais vers le français, c'est facile, vers l'allemand c'est facile, vers le russe aussi c'est facile parce que le fonds culturel commun est quasiment le même en fonction des conceptions culturelles. Mais en ce qui concerne la vision de l'indigène, c'est plus difficile. Ton expérience avec le guarani paraguayen n'est pas la meilleure pour cela parce qu'il s'agit d'une expérience avec une population d'origine espagnole. La langue est adaptée à la culture du peuple. Le guarani paraguayen n'est pas égal à celui du guarani du Kaiowá qui continue avec sa culture indigène moins influencée, moins partagée. Alors, la culture penche plus vers un côté, et des fois plus vers l'autre. Cela se répète dans l'histoire du monde de plusieurs régions. Une chose est l'arabe classique, l'autre chose est l'arabe de chaque pays.

AHR : Oui, c'est la situation des arabes dialectaux parlés dans différents pays en référence à l'arabe classique.

ADR : Parce qu'il y a différentes cultures, et les langues réflètent les différences culturelles. Langue/langage est un produit de la culture. En fonction de cela quand on change de territoire, alors on appelle cela différemment. On peut appeller cela une autre langue ou un autre dialecte. Et cette différence de langue et de dialecte est relative parce que si je dis que dans l'intérieur du Rio Grande do Sul on parle un dialecte gaucho - non pas le dialecte gaucho - c'est parce que le portugais parlé là-bas a des différences marquantes avec le portugais parlé à $500 \mathrm{~km}$ de là, dans le Paraná ou à São Paulo. On reconnaît le gaucho quand il parle, et je peux le montrer, il vient de cette région là-bas. On ne confond pas avec le paulista. Maintenant, c'est pareil de dire qu'il parle portugais, une variété de portugais, ou qu'il parle gauchês. Il n'y a pas de 
différence. Cela dépend de la situation culturelle. Tu as déjà eu une expérience avec le galicien ?

AHR : Oui, j'ai déjà entendu du galicien. À mon oreille, il est très proche du portugais.

ADR : Oui, il est très proche de l'espagnol. Le contraste est toujours avec d'autres langues. Toute cette terminologie est relative. Où commence le castillan, et où commence le catalan? Alors, du côté de l'Espagne, ils trouvent que le catalan est une variété de l'espagnol. Mais du côté de la France, ils trouvent que le catalan est une variété du français.

AHR : Oui, le point de vue change.

ADR : Justement parce qu'il y a des similitudes vers les deux côtés. Cette terminologie de langue et de dialecte est sujette à un haut degré d'arbitraire. Plusieurs fois ce sont des décisions politiques. La langue est nationale, d'une nation officialisée. Le reste est du dialecte. Il n'y a aucun critère objectif pour séparer langue et dialecte. Dialecte est nécessairement non compréhensible ? Il a la même origine, mais... Tout cela est arbitraire. Au Brésil, dire qu'il n'y a pas de dialecte ne fait aucun sens. Dialecte est la variété régionale d'une langue. Alors, avec cette aire immense qu'il y a, c'est rempli de dialectes. Quelquesuns plus fortement différenciés, d'autres moins fortement différenciés par rapport au modèle adopté par les intellectuels de la langue officielle.

AHR : Quel est le verbe que vous utiliseriez pour définir la traduction ? "Passer"? "Transmettre"?

ADR : Il y a un terme plus spécifique qui est celui de "traduire" qui sert pour tout degré de différence. Traduire des langues bien différentes comme le portugais-chinois, traduire du portugais vers l'espagnol, du catalan vers l'espagnol. "Traduire" a cet avantage. "Traduire" du chinois vers le portugais. On sait qu'il n'y a presque rien - superficiellement, tout du moins - en commun. Une phrase du chinois sera comprise par le locuteur de portugais. Mais elle sert aussi du catalan vers l'espagnol.

AHR : Vous considérez que superficiellement il n'y a pas de proximité entre le portugais et le chinois. Alors, vous trouvez qu'il existe un dénominateur commun?

ADR : Nécessairement.

AHR : C'est cela qui fait que la traduction soit possible?

ADR : Oui, c'est cela qui fait que la traduction soit possible qu'elle que soit la langue. Il n'y a pas de langue intraduisible pour n'importe quelle langue.

AHR : Dans ce cas, la fonction/rôle du traducteur est de trouver ce dénominateur? 
ADR : Le rôle du traducteur n'est pas de trouver quelque chose parce que le traducteur n'est pas un chercheur sur la nature de la langue. Mais il est un connaisseur des deux pratiques linguistiques. Il va trouver des correspondances pour les idées qui seront transmises dans une langue, pour passer dans l'autre langue. Il ne va pas penser comment fonctionne le verbe en chinois, quel est le système grammatical. Le traducteur n'a pas besoin de comprendre, ni d'analyser la langue comme le linguiste. Il doit connaître la langue. La plupart des gens qui traduit n'a rien appris de la grammaire, n'a aucune idée de comment cela fonctionne. Il suffit de dire les choses dans les deux langues. Par exemple, ici, au LALI nous avons plusieurs garçons. Il y en a un qui est polyglotte. Il a vécu avec d'autres langues. La tribu du père est kamayura qui est une espèce de capitale politique du Haut Xingu. Alors, là-bas, il y a toujours des mariages aussi bien avec de kamayura avec des indiennes non apparentées du point de vue linguistique, comme avec des indiennes kamayurá aussi. Ce sont les relations sociales. Dans la tribu kamayura, il a eu l'opportunité de rentrer en contact avec des indiens qui ont des langues vernaculaires différentes. Et ainsi il connaît quatre ou cinq langues indigènes. Non pas pour les étudier, mais simplement pour avoir vécu avec. Cela est normal, chose commune pour celui qui n'est pas scolarisé. Personne donne des cours de langue à personne. Tout le monde apprend la langue, vit avec et écoute ce que je parle en y entrant peu à peu. Et alors, peu importe quelle est la structure de la langue. Toutes sont des langues humaines. Toutes.

AHR : Vous avez parlé de correspondances. Comment est-ce que vous réglez des concepts et des situations qui n'existent pas dans une autre langue en termes culturels, de représentation de la société ?

ADR : S'il domine les deux langues, quand il n'y a pas la rencontre de conceptions basiques, ce qu'il fait est simplement de décrire la langue qu'il utilise vers laquelle il traduit, une traduction plus ou moins littérale de ce que les autres disent. Alors il doit comprendre un peu comme interprète, dire qu'ils comprennent comme ça même si ce n'est pas ce qu'il a appris. Il doit avoir les pieds des deux côtés, du côté de la langue de l'autre, et de la langue vers laquelle il traduit.

AHR : Vous établissez une différence entre traducteur et interprète ?

ADR : Oui. Vous pouvez distinguer la traduction comment étant plus directe. $\mathrm{Tu}$ établis les correspondances avec des situations semblables. En portugais, on dit : "O sol nasce às 6 horas". En anglais, "The sun appeared at 6 o'clock." "Appeared" n'est pas le même que "nasceu." Tu ne traduis pas au pied de la lettre parce que sinon ça ne sort pas en anglais. Tu adaptes spécialement la traduction versus l'interprétation. On essaie de faire que le "nascer" du portugais devienne le "aparecer-appear" de l'anglais : "O sol nasce." 
AHR : Dans le cas de cet exemple du soleil, ce sont des conceptions relativement proches. J'ai un exemple en tête en rapport avec le guarani paraguayen. Il a de nombreux mots pour dire la lumière du soleil quand le soleil naît. Ce moment du matin a plusieurs mots pour dire l'intensité de la lumière, et métaphoriquement du soleil qui naît et du jour qui arrive. En portugais, nous n'avons pas cette variété. Dans un cas comme celui-ci, que devient la traduction? Nous n'avons pas non plus en français. On dit "le lever du jour", mais cela n'exprime pas l'idée. Même ce mot "madrugada" n'existe pas. Il y a "le lever du jour", "le matin", mais cet espace de temps si diversifié n'existe pas en français. Dans le cas du guarani et du portugais, comment faire cette traduction avec ces nuances toutes culturelles ? Alors, je n'aurai pas comment traduire?

ADR : Tu vas expliquer. Comment tu vas donner l'idée à l'autre de ce qui a été dit de ce côté-ci? La traduction n'est pas une chose comme ça aussi directe. Cela dépend des contextes dans lequel cette langue sera utilisée, et ce sont des contextes culturels. Le fait de distinguer en guarani plus de phases différentes dans l'espace du matin, cela est une chose naturelle. Cela ne change pas la réalité, c'est une segmentation de la réalité. Et tu peux dire en portugais des choses comme "ele madrugou". Comment tu dis "ele madrugou" en français?

AHR : "Il s'est levé tôt.", "il s'est réveillé tôt." Mais cela signifie qu'il "madrugou/ s'est levé tôt.

ADR : Cela ne donne pas l'idée de "madrugada"/[petit matin]. Mais ce terme n’a pas seulement à voir avec "acordar"/[se réveiller], mais aussi avec "n'a pas dormi". "Ele madrugou fazendo tal coisa...". "Ele não dormiu", par conséquent, "il n'a pas fermé l'œil". Ce sont des choses d'intérêt culturel, ce ne sont pas des différences linguistiques. Alors, la traduction est essayer de passer ce qui est exprimé dans une langue vers l'autre mais en prenant en compte les différences culturelles. Alors, la traduction n'est pas seulement d'une langue vers une autre, mais d'une culture vers une autre. Le traducteur qui connaît la langue et ne connaît pas la culture, n'arrive pas à traduire comme il faut, sauf des choses superficielles. Comment traduire un philosophe chinois sans connaître la culture chinoise, la culture chinoise ? C'est impossible.

AHR : Vous considérez qu'un des travaux que le traducteur doit faire est de situer ce matériel culturel dans la culture. D'où vient ce qui est l'objet de la traduction?

ADR : Oui, la formation du traducteur devrait être beaucoup plus que linguistique, mais culturelle, sociologique et anthropologique. Sans connaître la culture, il n'y a pas de conditions de faire une bonne traduction. La traduction littérale donne alors une bonne compréhension. 


\title{
Kaingang: gender or classifiers?
}

\author{
Wilmar da Rocha D’Angelis ${ }^{1}$
}

Le genre grammatical est l'une des categories grammaticales les moins logiques et le plus innattendue.

(A. Meillet 1965:202) ${ }^{2}$

The linguistic system, yet stripped from "reason" (or by force be stripped from reason) always speaks to imagination and drives it.

(L. Hjemslev 1971:227)

\begin{abstract}
The Kaingang language presents a particular vocalic alternation involving low nasal vowels, which has traditionally been treated either as dialect differences or as a case of "free variation". My two-decade contact with native speakers of several Kaingang communities - particularly from Xapecó (SC), Nonoai, and Inhacorá (RS) - allows me to consider this vocalic alternation as a kind of "classifier" with some characteristics of "gender". This paper argues that these distinctions in pronunciation are not mere dialect differences in Kaingang. The linguistic reality is much richer, more revealing and more interesting than the magical discard of "free variation".
\end{abstract}

\section{Introduction}

The Kaingang language presents a particular vocalic alternation involving low nasal vowels, which has traditionally been treated either as dialect

\footnotetext{
1 Doctor in Linguistics and indigenist, Professor at the Linguistics Department of Unicamp (Campinas, SOP - Brazil)

2 "Grammatical gender is one of the least logical and more unexpected grammatical categories".
} 